\title{
The JUNO Calibration System
}

\author{
Qingmin Zhang', Yuhang Guo \\ Department of Nuclear Science and Technology, School of Energy and Power Engineering, Xi'an \\ Jiaotong University, Xi'an 710049, China \\ E-mail:zhangqingminemail.xjtu.edu.cn, guoyuhangestu.xjtu.edu.cn
}

\section{On behalf of JUNO collaboration}

The Jiangmen Underground Neutrino Observatory (JUNO) is designed to primarily measure the neutrino Mass Hierarchy. Its Central Detector (CD) will be the largest liquid scintillator (LS) detector to measure the energy of neutrinos with an unprecedented energy resolution of 3\% ( $1 \mathrm{MeV}$ and an energy nonlinearity better than $1 \%$. Accordingly, a calibration complex is designed for multiple source deployment, the energy coverage of reactor neutrinos and CD fullvolume coverage. In this proceeding, the design details and primary progress about JUNO calibration system are presented.

The 39th International Conference on High Energy Physics (ICHEP2018)

4-11 July, 2018

Seoul, Korea 


\section{Introduction of JUNO}

The Jiangmen Underground Neutrino Observatory (JUNO) is designed to primarily determine the neutrino Mass Hierarchy(MH) and to discover other physics with energy resolution better than $3 \% / \sqrt{\mathrm{E}}$ [1] . JUNO central detector (CD), an acrylic sphere with a diameter of $35.4 \mathrm{~m}$, is filled with liquid scintillator(LS) to measure the energy of neutrinos ${ }^{[2]}$, so the calibration system is very critical ${ }^{[3,4,5]}$.

\section{Calibration System}

JUNO energy response is strongly position-dependant, so a complete calibration complex including Automatic Calibration Unit (ACU), Cable Loop System (CLS), Guide Tube Control System(GTCS) and Remotely Operated under-liquid-scintillator Vehicles (ROV) has been designed for full coverage ${ }^{[6]}$.

The radiation sources include neutron sources $\left({ }^{241} \mathrm{Am}-\mathrm{Be},{ }^{241} \mathrm{Am}-{ }^{13} \mathrm{C},{ }^{241} \mathrm{Pu}-{ }^{13} \mathrm{C},{ }^{252} \mathrm{Cf}\right)$, positron sources $\left({ }^{22} \mathrm{Na},{ }^{68} \mathrm{Ge},{ }^{40} \mathrm{~K},{ }^{90} \mathrm{Sr}\right)$ and gamma sources $\left({ }^{40} \mathrm{~K},{ }^{54} \mathrm{Mn},{ }^{60} \mathrm{Co},{ }^{137} \mathrm{Cs}\right)$ will be used for calibration ${ }^{[6]}$.

Table 1 key features of calibration sub-systems

\begin{tabular}{|c|c|c|c|c|c|}
\hline Sub-system & Frequency & Positioning & Position Control & Positioning Error & Source change \\
\hline $\mathrm{ACU}$ & Weekly & Rope Length & \multirow{3}{*}{$\begin{array}{l}\text { Spool drive (steel } \\
\text { wire coated with } \\
\text { Teflon } \Phi 1.0 \text { ) } \\
+ \text { Tension Control }\end{array}$} & a few mm & Manual \\
\hline CLS & Monthly & $\begin{array}{l}\text { Rope Length, CCD } \\
\text { Ultrasonic receiver }\end{array}$ & & $\begin{array}{c}10 \mathrm{~cm} \text { (rope) } \\
3 \mathrm{~cm} \text { (w/ sensors) }\end{array}$ & Automatic \\
\hline GTCS & Monthly & $\begin{array}{l}\text { Rope Length } \\
\text { Metal Sensor }\end{array}$ & & $\begin{array}{c}10 \mathrm{~cm} \text { (rope) } \\
3 \mathrm{~cm}(\mathrm{w} / \text { sensors })\end{array}$ & Manual \\
\hline ROV & $\begin{array}{c}\text { When needed, } \\
\text { seasonally or } \\
\text { annually }\end{array}$ & $\begin{array}{l}\text { Ultrasonic receiver } \\
\text { CCD }\end{array}$ & $\begin{array}{c}\text { Remotely Operated } \\
\text { Vehicle }\end{array}$ & $\sim 4 \mathrm{~cm}$ & Manual \\
\hline
\end{tabular}

\section{Calibration Strategy}

ACU, CLS and GTCS will be combined to deliver ${ }^{40} \mathrm{~K}$ source to specified positions in one plane for non-uniformity correction, and ROV will be used for full volume scan when needed. On the other hand, ACU with multiple sources at CD center will be used for nonlinearity correction. Finally, the energy can be measured accurately.

JUNO response function can be calibrated by using the data from the given calibration points with ACU, CLS and GTCS (or ROV). A simple spline function is used to predicate the "blank" region and the energy response uniformity would be corrected with the correction function. Simulation shows that energy resolution for $1.022 \mathrm{MeV}$ uniformly distributed positron is $2.98 \%$ and that the bias of the mean value is $\sim 0.04 \%{ }^{[7]}$.

Energy linearity is corrected by placing various sources at CD center with ACU. 9 gamma sources are used to study the detector's linearity. Verification shows that the energy bias (non-linearity) to monoenergy positron at $\mathrm{CD}$ center is $<0.5 \%$ after correction ${ }^{[7]}$.

The simulated mono-energy e $\mathrm{e}^{+}$events are uniformly distributed in $\mathrm{CD}$ and the non-uniformity correction (response is obtained with ${ }^{40} \mathrm{~K}$ ) is applied. Analysis shows that the bias is less than $0.1 \%$ and the energy resolution is better than $3.0 \%{ }^{[6]}$, which satisfies the JUNO requirements.

\section{References}

[1] F.P. An et al., Neutrino physics with JUNO [J], J. Phys. G: Nucl. Part. Phys. 43 (2016) 030401 (188pp).

[2]T. Adam et al., JUNO Conceptual Design Report [R], https://arxiv.org/abs/1508.07166.

[3] L. Zhan, Y.F. Wang, Determination of the neutrino mass hierarchy at an intermediate baseline [J], Phys. Rev. D 78, 111103 (2008).

[4] L. Zhan, Y.F. Wang, Experimental requirements to determine the neutrino mass hierarchy using reactor neutrinos [J], Phys. Rev. D 79, 073007 (2009).

[5] Y.F. Li, J. Cao, Unambiguous determination of the neutrino mass hierarchy using reactor neutrinos [J], Phys. Rev. D 88, 013008 (2013).

[6] Q.M. Zhang et al. , JUNO Central Detector and its Calibration System [J], $38^{\text {th }}$ International Conference on High Energy Physics, Chicago, USA,3-10 August,2016, Proceedings of Science, PoS(ICHEP2016)967.

[7] F.Y. Zhang, Calibration for energy non-linearity and resolution [R], Beijing: IHEP, 2018. 